Mariana Oliveira de Alencar Ramalho ${ }^{1}$

Paulo Germano de Frias ${ }^{2}$

Lygia Carmen de Moraes Vanderlei ${ }^{1}$

Vilma Costa de Macêdo ${ }^{3}$

Pedro Israel Cabral de Lira ${ }^{4}$

${ }^{1}$ Programa de PósGraduação em Saúde da Criança e do Adolescente, Universidade Federal de Pernambuco. Av Professor Morais Rego $\mathrm{s} / \mathrm{n}$, Cidade Universitária. 50670-420 Recife PE Brasil.mariramalho_24@ hotmail.com

${ }^{2}$ Grupo de Estudos em Avaliação em Saúde, Instituto de Medicina Integral Professor Fernando Figueira.

${ }^{3}$ Departamento de Enfermagem, Universidade Federal de Pernambuco (UFPE).

${ }^{4}$ Departamento de Nutrição, UFPE.

\section{Avaliação da incompletude da declaração de óbitos de menores de um ano em Pernambuco, Brasil, 1999-2011}

\author{
Evaluation of the incompleteness in the filling out \\ of death certificates of children under one year of age \\ in the State of Pernambuco, Brazil, 1999-2011
}

Abstract In order to assess the incompleteness in filling out death certificates (DC) in the regional health offices of the State of Pernambuco a descriptive study was conducted on data for infant mortality information on deaths of children under one year of age in the municipalities of Pernambuco in the 1999-2001 and 2009-2011 threeyear periods. The percentage of incompleteness was based on the blank/unknown responses in the DCs per regional health office and the proportional variation rate of incompleteness between the two periods. It was observed that the filling out of information did not show a well-defined pattern per office, with a reduction in incompleteness occurring for most variables, though the sex, age and place of occurrence of death variables alone revealed $\leq 5 \%$ incompleteness in the second term of study, which was considered excellent in terms of the Romero and Cunha scores. Despite the decrease in incompleteness related to pregnancy, childbirth and mother-related variables, in the second triennium incompleteness between 10 and $20 \%$ was observed, with a regular score in the 2009-2011 triennium. Despite the improvement in the filling out of details in the State, it is necessary to strengthen surveillance and training for completing the DCs by the Committees for Prevention of Infant Mortality.

Key words Infant mortality, Information systems, Vital statistics
Resumo Com o objetivo de avaliar a incompletude no preenchimento da declaração de óbito (DO) nas Regionais de Saúde do estado de Pernambuco foi realizado um estudo descritivo a partir de informações sobre óbitos de menores de um ano residentes dos municípios de Pernambuco, provenientes do Sistema de Informação sobre Mortalidade, nos triênios de 1999-2001 e 2009-2011. Foi calculado o percentual de incompletude considerando o preenchimento em branco/ignorado dos campos da DO por Regional de Saúde e a taxa de variação proporcional da incompletude entre os dois triênios. Observou-se que o preenchimento não apresentou um padrão bem definido para as Regionais, ocorrendo redução da incompletude para a maioria das variáveis, entretanto, apenas as variáveis sexo, faixa etária e local de ocorrência do óbito atingiram incompletude $\leq 5 \%$ no segundo triênio de estudo, considerado excelente nos escores de Romero e Cunha. Apesar do decréscimo da incompletude das variáveis relacionadas à gestação e parto e relacionadas à mãe, foi observado no segundo triênio incompletude entre 10 e 20\%, atingindo escore regular no triênio 2009-2011. Apesar da melhoria do preenchimento no Estado, faz-se necessário o fortalecimento da vigilância e dos Comitês de Prevenção do Óbito Infantil para qualificação do preenchimento da DO.

Palavras-chave Mortalidade infantil, Sistemas de informação, Estatísticas vitais 


\section{Introdução}

A informação exerce um importante papel no planejamento de políticas de saúde e na tomada de decisões, provendo evidências epidemiológicas sobre o perfil sanitário que proporciona o reconhecimento das necessidades de saúde da população ${ }^{1,2}$.

Até a década de 1970 o Brasil não possuía um padrão para o registro de dados referentes aos eventos vitais. Os dados sobre mortalidade eram pouco abrangentes e não confiáveis, e eram gerados nos cartórios de registro civil, que possuíam na época mais de 40 modelos de atestados de óbito sem padronização destes documentos ou do seu fluxo ${ }^{1}$.

Apenas em 1975 com o advento do Sistema de Vigilância Epidemiológica do Ministério da Saúde (MS), foi criado o Sistema de Informação sobre Mortalidade (SIM), que introduziu o modelo único da Declaração de Óbito (DO) proposto pela Organização Mundial de Saúde (OMS) com fluxos padronizados para todo o País ${ }^{1}$.

Atualmente, a DO é impressa em três vias de cores distintas e numerada pelo MS, dividida em nove blocos, sendo o IV referente aos óbitos em menores de um ano, que inclui variáveis como peso ao nascer, sexo e duração da gestação. O preenchimento dessas variáveis de forma correta e completa permite melhor compreensão do evento, propiciando intervenções aos grupos mais vulneráveis ao óbito infantil ${ }^{1,3}$.

Para que o SIM subsidie as decisões no campo da saúde coletiva adequadas à realidade local para a melhoria da assistência materno infantil, é necessário que possua boa cobertura, regularidade e preenchimento adequado de todos os campos da DO, com redução de informações ignoradas ou em branco e das inconsistências ${ }^{4,5}$.

Estudos nacionais apontam para a melhoria da adequação das informações vitais sobre nascidos vivos e óbitos nas últimas décadas ao analisar indicadores de cobertura, regularidade e percentual de causas mal definidas de óbito ${ }^{6,7}$. Igualmente, estudos relacionados à confiabilidade $\mathrm{e}$ completude dos campos da DO mostram variações na consistência e no grau de preenchimento da informação na dependência do grupo etário e causa básica de morte ${ }^{8,9}$. Em relação aos óbitos infantis, mesmo aqueles ocorridos em unidades hospitalares, são descritos problemas relacionados à qualidade do registro ${ }^{10,11}$.

Nos últimos anos o MS tem estimulado a realização de pesquisas para avaliar as informações vitais, em particular sobre óbitos infantis ${ }^{12-14}$. A análise da DO de menores de um ano pode apontar localidades que precisam de investimentos para superar as fragilidades encontradas em relação às informações ${ }^{15}$. Adicionalmente, a qualidade das informações sobre óbitos infantis expressam em alguma medida as iniquidades na assistência à saúde de grupos mais vulneráveis, particularmente as barreiras de acesso aos serviços ${ }^{16}$.

Apesar dos avanços na cobertura dos Sistemas de Informação sobre eventos vitais e na ampliação da rede assistencial, o estado de Pernambuco permanece com lacunas na produção dos dados ${ }^{17}$. Desta forma, este estudo pretende avaliar comparativamente a incompletude no preenchimento das variáveis da DO de menores de um ano residentes em Pernambuco em dois triênios da década de 2000.

\section{Método}

Trata-se de estudo epidemiológico descritivo que utilizou os dados sobre óbitos de menores de um ano provenientes do SIM referentes aos residentes em todos os municípios de Pernambuco, nos triênios 1999-2001 e 2009-2011, disponíveis na página do Datasus ${ }^{18}$. Foram utilizados os dados trienais com o objetivo de dar maior estabilidade aos indicadores.

A análise é apresentada por Regional de Saúde (RS) de Pernambuco, que totalizam 12, distribuídas em uma área de $98.148,323 \mathrm{~km}^{2}$, contendo 8.786.448 habitantes (hab. ${ }^{19}$. As RS apresentam número de habitantes e municípios diferenciados. A I Regional contempla 18 municípios e a Ilha de Fernando de Noronha, com 3.964.806 habitantes; a II, 22 municípios e 573.337 hab.; a III, 22 municípios e 582.870 hab.; a IV, 32 municípios e 1.266.476 hab.; a V, 21 municípios e 518.427 hab.; a VI, 13 municípios e 390.913 hab.; a VII, 7 municípios e 140.295 hab.; a VIII, 7 municípios e 448.780 hab.; a IX, 11 municípios e 332.530 hab.; a X, 12 municípios e 182.015 hab.; a XI, 10 municípios e 226.493 hab.; e a XII, 10 municípios e 304.086 hab. ${ }^{18}$.

O banco de dados construído continha variáveis relativas aos óbitos infantis que foram classificadas em quatro grupos: as relacionadas à criança (sexo, cor/raça, peso ao nascer, faixa etária); à gestação (duração da gravidez, tipo de gravidez, tipo de parto); à mãe (idade e escolaridade) e ao momento do óbito (local de ocorrência, óbito em relação ao parto).

Cada variável foi tabulada anualmente a cada triênio e, posteriormente, foi realizado o cálculo 
do percentual de incompletude considerando o preenchimento em ignorado ou em branco dos campos da DO de menores de um ano por RS de residência nos triênios considerados. Para cada uma das regionais foi calculada a taxa de variação proporcional da incompletude entre os dois triênios. Adicionalmente, foi calculada a incompletude das mesmas variáveis para o Nordeste e Brasil além da taxa de variação para comparação entre os achados.

Para classificação da incompletude foram utilizados os escores propostos por Romero e Cunha ${ }^{20}$ : excelente $(<$ de $5 \%$ de preenchimento incompleto); bom (5 a $<10 \%)$; regular $(10 \mathrm{a}<20 \%)$; ruim $(20 \mathrm{a}<50 \%)$ e muito ruim ( $50 \%$ ou mais).

O referido estudo foi submetido e aprovado pelo Comitê de Ética em Pesquisa do Centro de Pesquisa Aggeu Magalhães (CPqAM) da Fundação Oswaldo Cruz.

\section{Resultados}

Na Tabela 1 apresentam-se os dados de incompletude para o primeiro e segundo triênios do estudo (1999-2001 e 2009-2011). Em relação ao primeiro triênio as variáveis sexo, faixa etária $e$ local de ocorrência do óbito foram as únicas que apresentaram escore excelente $(<5 \%)$ para regionais e estado. Os maiores percentuais de incompletude encontrados foram referentes às variáveis relacionadas à gestação e parto e à mãe. Destacam-se a II e a V RS que apresentaram incompletude acima de 50\% para estas variáveis, considerado preenchimento muito ruim. Em contrapartida a VI RS teve o menor percentual para as essas variáveis, entre $12,4 \%$ para tipo de gravidez e $15 \%$ para escolaridade da mãe. A variável óbito em relação ao parto obteve escore ruim ou muito ruim em dez das doze regionais, apenas as I e VII RS atingiram escore regular.

Ainda na Tabela 1, no segundo triênio de estudo (2009-2011), para as RS e estado, o percentual de incompletude de preenchimento das variáveis sexo, faixa etária e local de ocorrência do óbito comportaram-se de forma igual ao primeiro triênio. A variável cor/raça atingiu escore excelente de preenchimento nas II, IV, VI e XI $\mathrm{RS}$, apresentando incompletude menor do que a encontrada para o estado no mesmo período. Para a variável peso ao nascer a maior parte das RS seguiu a tendência observada no estado, com preenchimento regular. Dentre as variáveis relacionadas à gestação e parto, no segundo triênio houve redução da incompletude das variáveis tipo de gravidez e tipo de parto nas I, IX, X RS, que atingiram escore bom de preenchimento $(5 \mathrm{a}<$ $10 \%)$. Apesar da redução da incompletude, a V RS apresentou-se como a de pior preenchimento das variáveis relacionadas à gestação e parto e à mãe, com escore ruim.

A Tabela 2 mostra a taxa de variação proporcional da incompletude entre os dois triênios de estudo. A maior parte das variáveis atingiu redução na incompletude nas RS e estado, com destaque para as variáveis cor/raça e óbito em relação parto que obtiveram redução de $77,4 \%$ e $62,6 \%$, respectivamente. Observou-se redução da incompletude das variáveis relacionadas à gestação e parto nas RS e estado, com exceção das III, VI e X RS onde ocorreram incrementos de $30,8 \%$, $55,4 \%$ e $24 \%$, respectivamente, na incompletude da variável duração da gestação. No grupo de variáveis relacionadas à mãe também ocorreu redução da incompletude na maioria das regionais, apenas a VI apresentou incremento de 3,4\% na idade e $5,7 \%$ na escolaridade.

A Tabela 3 apresenta os percentuais e a taxa de variação proporcional da incompletude de preenchimento das variáveis da DO para o Brasil, Nordeste e Pernambuco. As variáveis sexo, faixa etária e local de ocorrência do óbito, assim como nas RS, foram as únicas que atingiram escore excelente nos dois períodos no Nordeste e Brasil. O estado de Pernambuco destaca-se no preenchimento da variável cor/raça, atingindo um escore bom no segundo triênio, com 7,4\% de incompletude, superando o alcançado no Brasil e Nordeste.

O preenchimento das variáveis relacionadas à gestação e parto em Pernambuco foi semelhante ao observado no Brasil e Nordeste, com redução da incompletude e escore regular de preenchimento no segundo triênio. A variável escolaridade da mãe foi mais preenchida em Pernambuco do que no Brasil e Nordeste, atingindo escore regular em 2009-2011, com redução de 58,9\% de incompletude.

\section{Discussão}

Nos dois triênios de estudo o preenchimento das variáveis da DO de menores de um ano não apresentou um padrão bem definido para as RS, e apesar de haver ocorrido uma redução da incompletude para a maioria delas, poucas atingiram um preenchimento excelente.

A presente análise corrobora com resultados encontrados em outros estudos com relação à re- 


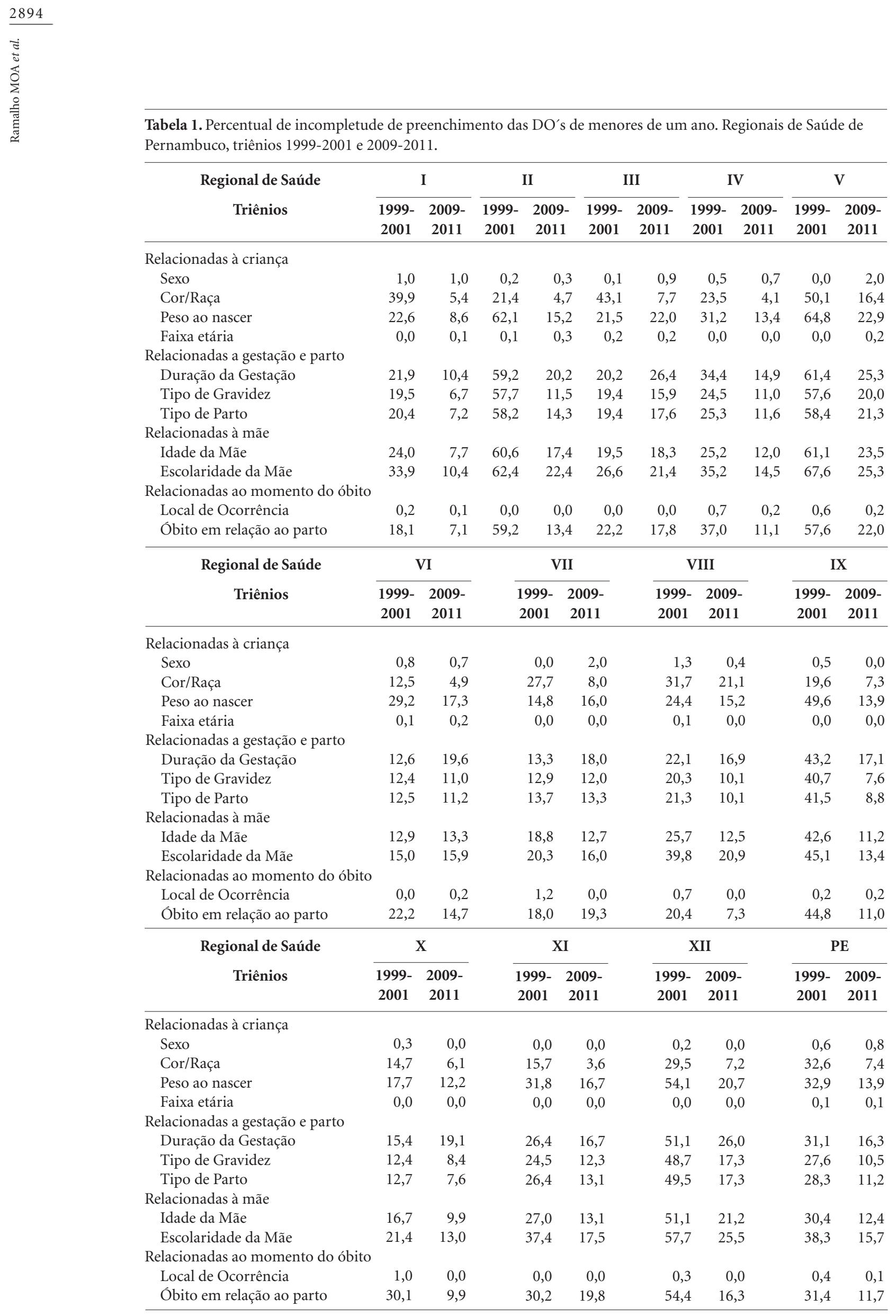

Fonte: SIM/Datasus/MS. 
Tabela 2. Taxa de variação proporcional da incompletude de preenchimento das DO de menores de um ano. Regionais de Saúde de Pernambuco, triênios 1999-2001 e 2009-2011.

\begin{tabular}{|c|c|c|c|c|c|c|c|c|c|c|c|c|c|}
\hline Regional de Saúde & I & II & III & IV & $\mathbf{V}$ & VI & VII & VIII & IX & $\mathrm{X}$ & XI & XI & PE \\
\hline \multicolumn{14}{|l|}{ Relacionadas à criança } \\
\hline Sexo & $-3,3$ & 39,6 & 800,0 & 26,4 & 0,0 & $-7,5$ & $-65,8$ & $-65,8$ & $-100,0$ & $-100,0$ & 0,0 & $-100,0$ & 34,0 \\
\hline Cor/Raça & $-86,6$ & $-78,2$ & $-82,1$ & $-82,7$ & $-67,3$ & $-60,7$ & $-33,4$ & $-33,4$ & $-62,7$ & $-58,5$ & $-77,3$ & $-75,5$ & $-77,4$ \\
\hline Peso ao nascer & $-62,1$ & $-75,5$ & 2,4 & $-57,0$ & $-64,7$ & $-40,7$ & $-37,8$ & $-37,8$ & $-72,0$ & $-31,1$ & $-47,5$ & $-61,8$ & $-57,7$ \\
\hline Faixa etária & 5,5 & 179,2 & $-6,8$ & $-100,0$ & 0,0 & 85,0 & $-100,0$ & $-100,0$ & 0,0 & 0,0 & 0,0 & 0,0 & 77,8 \\
\hline \multicolumn{14}{|l|}{ Relacionadas a gestação e parto } \\
\hline Duração da Gestação & $-52,6$ & $-65,9$ & 30,8 & $-56,6$ & $-58,7$ & 55,4 & $-23,5$ & $-23,5$ & $-60,5$ & 24,0 & $-36,9$ & $-49,2$ & $-47,4$ \\
\hline Tipo de Gravidez & $-65,4$ & $-80,1$ & $-18,4$ & $-55,1$ & $-65,4$ & $-11,3$ & $-50,1$ & $-50,1$ & $-81,4$ & $-32,1$ & $-49,8$ & $-64,5$ & $-62,0$ \\
\hline Tipo de Parto & 54,6 & $-75,4$ & $-9,0$ & $-54,0$ & $-63,5$ & $-10,3$ & $-52,4$ & $-52,4$ & $-78,8$ & $-39,9$ & $-50,4$ & $-65,0$ & $-60,3$ \\
\hline \multicolumn{14}{|l|}{ Relacionadas à mãe } \\
\hline Idade da Mãe & $-67,9$ & $-71,3$ & $-6,2$ & $-52,5$ & $-61,5$ & 3,4 & $-51,2$ & $-51,2$ & $-73,7$ & $-40,7$ & $-51,6$ & $-58,6$ & $-59,0$ \\
\hline Escolaridade da Mãe & $-69,3$ & $-64,2$ & $-19,6$ & $-58,9$ & $-62,5$ & 5,7 & $-47,5$ & $-47,5$ & $-70,3$ & $-39,4$ & $-53,3$ & $-55,8$ & $-58,9$ \\
\hline \multicolumn{14}{|l|}{$\begin{array}{l}\text { Relacionadas ao momento do } \\
\text { óbito }\end{array}$} \\
\hline Local de Ocorrência & $-32,7$ & 0,0 & 0,0 & $-69,0$ & $-59,8$ & 0,0 & $-100,0$ & $-100,0$ & 52,9 & $-100,0$ & 0,0 & $-100,0$ & $-61,2$ \\
\hline Óbito em relação ao parto & $-60,5$ & $-77,4$ & $-19,7$ & $-70,1$ & $-61,9$ & $-33,8$ & $-64,4$ & $-64,4$ & $-75,5$ & $-67,0$ & $-34,3$ & $-70,0$ & $-62,6$ \\
\hline
\end{tabular}

Fonte: SIM/Datasus/MS.

Tabela 3. Percentual e taxa de variação proporcional da incompletude de preenchimento das DO de menores de um ano. Brasil, Nordeste e Pernambuco, 1999-2001 e 2009-2011.

\begin{tabular}{|c|c|c|c|c|c|c|c|c|c|}
\hline \multirow[b]{3}{*}{ Relacionadas à criança } & \multicolumn{3}{|c|}{ Brasil } & \multicolumn{3}{|c|}{ Nordeste } & \multicolumn{3}{|c|}{ Pernambuco } \\
\hline & 1999-2001 & 2009-2011 & tx var & 1999-200 & 2009-2011 & 1 txvar & 1999-2001 & 2009-2011 & tx var \\
\hline & & & & & & & & & \\
\hline Sexo & 0,7 & 0,5 & $-31,7$ & 1,4 & 0,8 & $-44,8$ & 0,6 & 0,8 & 34,0 \\
\hline Cor/Raça & 32,3 & 12,0 & $-62,8$ & 48,7 & 19,3 & $-60,3$ & 32,6 & 7,4 & $-77,4$ \\
\hline Peso ao nascer & 43,5 & 16,2 & $-62,8$ & 52,7 & 18,0 & $-65,9$ & 32,9 & 13,9 & $-57,7$ \\
\hline Faixa etária & 0,1 & 0,1 & $-49,7$ & 0,2 & 0,1 & $-66,8$ & 0,1 & 0,1 & 77,8 \\
\hline Relacionadas a gestação e part & & & & & & & & & \\
\hline Duração da Gestação & 42,2 & 17,3 & $-58,9$ & 47,8 & 20,3 & $-57,6$ & 31,1 & 16,3 & $-47,4$ \\
\hline Tipo de Gravidez & 38,8 & 12,8 & $-66,9$ & 44,1 & 13,8 & $-68,7$ & 27,6 & 10,5 & $-62,0$ \\
\hline Tipo de Parto & 38,5 & 13,7 & $-66,9$ & 44,6 & 14,7 & $-67,0$ & 28,3 & 11,2 & $-60,3$ \\
\hline Relacionadas à mãe & & & & & & & & & \\
\hline Idade da Mãe & 42,0 & 17,7 & $-57,9$ & 48,7 & 19,1 & $-60,8$ & 30,4 & 12,4 & $-59,0$ \\
\hline Escolaridade da Mãe & 51,6 & 24,5 & $-52,5$ & 58,7 & 26,3 & $-55,2$ & 38,3 & 15,7 & $-58,9$ \\
\hline Relacionadas ao momento do & & & & & & & & & \\
\hline Local de Ocorrência & 3,4 & 0,1 & $-95,9$ & 3,4 & 0,2 & $-94,7$ & 0,4 & 0,1 & $-61,2$ \\
\hline Óbito em relação ao parto & 61,6 & 16,4 & $-73,4$ & 58,6 & 17,1 & $-70,8$ & 31,4 & 11,7 & $-62,6$ \\
\hline
\end{tabular}

Fonte: SIM/Datasus/MS.

dução da incompletude das variáveis sexo, faixa etária da criança e local de ocorrência ${ }^{10,20-22}$, atingindo escore excelente. Para Romero e Cunha ${ }^{20}$ a qualidade no preenchimento de variáveis socioeconômicas e demográficas proporciona melhor monitoramento e planejamento de ações e serviços capazes de evitar o óbito infantil.

Apesar do incremento observado no preenchimento da variável escolaridade da mãe, esta apresentou o maior número de regionais com incompletude superior a $20 \%$ nos dois triênios. Estudo realizado em Pernambuco utilizando o linkage dos bancos de dados do SIM e Sinasc identificou que esta foi a variável menos preenchida no SIM, atingindo $16,5 \%$ de incompletude ${ }^{21}$.

De forma igual, pesquisa sobre óbitos neonatais precoces em Ribeirão Preto $(\mathrm{SP})^{23}$, mostrou que a escolaridade materna foi a variável menos 
preenchida com $40 \%$ de omissão. No mesmo estudo a idade materna apresentou $20 \%$ de ignorados/bancos e o peso ao nascer $12,4 \%$, enquanto em Pernambuco no segundo triênio, o percentual de incompletude para idade materna e peso ao nascer alcançou os valores de $12,4 \%$ e de quase $14 \%$, respectivamente.

Salienta-se que mesmo em estudos sobre mortalidade neonatal precoce desenvolvidos nas Regiões Nordeste e Sudeste ${ }^{23,24}$, com eventos ocorridos em ambiente hospitalar, muito próximos ao parto com maior possibilidade de resgate de informações para preenchimento da DO com a família e prontuário, houve um percentual muito elevado de incompletude.

A duração da gestação apresentou o pior preenchimento entre as variáveis relacionadas à gestação e parto, o que ratifica achados de outros estudos $^{10,21-24}$. Chama a atenção o fato de que o elevado percentual de variáveis ignoradas/em branco podem alterar o perfil da mortalidade infantil, a exemplo do que já foi demonstrado com relação à cobertura incompleta em estudos realizados no município de Olinda $(\mathrm{PE})^{25}$, onde, através da busca ativa de óbitos não informados ao SIM, constataram-se alterações no coeficiente de mortalidade infantil e no perfil epidemiológico, inclusive da causa básica do óbito? .

O acúmulo de deficiências no SIM, como: a cobertura incompleta do sistema; a irregularidade da informação; os óbitos com causa mal definida ou pouco especificada; a alta proporção de preenchimento ignorado e/ou em branco associados a problemas na consistência e confiabilidade das informações, limita o seu uso na compreensão dos fatores de risco envolvidos na mortalidade infantil e no planejamento de estratégias para prevenção desse evento ${ }^{22}$.

Em Pernambuco, as RS com melhores resultados são coincidentes com as que dispõem de maior acesso aos serviços, rede assistencial mais organizada e vigilância atuante com atividades de resgate de variáveis, investigação dos óbitos e capacitações regulares dos profissionais de saúde, em geral localizadas mais próximas da capital.

Diversos autores apontam entraves na geração, consolidação e divulgação das informações sobre mortalidade. Destacam-se a pouca importância dada ao preenchimento adequado da DO pelo médico, encarando-o apenas como documento de valor legal e burocrático ${ }^{26,27}$; o preenchimento da DO delegado a profissionais administrativos $^{11,28}$; mau preenchimento do prontuário médico ${ }^{10}$; a pouca clareza nas instruções de preenchimento preconizadas pelo $\mathrm{MS}^{20,29}$ e a falha na digitação dos dados da DO para o sistema ${ }^{24}$.

Além disto, o hiato de dois anos existente entre a ocorrência do óbito e a divulgação do dado através da página do Datasus, representa uma deficiência para o monitoramento e vigilância do óbito infantil ${ }^{20}$. Igualmente, o preenchimento priorizado de determinadas variáveis como tipo de óbito e causa básica, talvez decorrente de classificação que categorizava as variáveis em indispensáveis, essenciais e secundárias ${ }^{11}$.

Em oposição, pouco antes do primeiro triênio analisado, com a implantação do óbito infantil como evento sentinela, houve melhoria no preenchimento e confiabilidade das informações, constituindo-se em um dos investimentos dos entes federados para minimizar os entraves relacionados à qualidade do preenchimento da DO de menores de um ano ${ }^{4}$. Os Comitês de Prevenção do Óbito Infantil e os Grupos Técnicos de discussão dos casos, institucionalizados a partir de 1999 como estratégias para monitorar e reduzir a mortalidade infantil por causas evitáveis contribuíram para melhorar a adequação das informações vitais ${ }^{30}$. Mais recentemente, o MS lançou o Painel de Monitoramento da Mortalidade Infantil e Fetal, o qual permite acompanhar com maior agilidade as informações sobre óbitos infantis e fetais mensalmente ${ }^{31}$.

Contudo, o incremento na completude e na consistência das informações só será possível se houver um maior compromisso municipal na correção do banco de dados do SIM após a conclusão da investigação do óbito. A incorporação da análise da completude da DO nas avaliações da adequação das informações ao agregar a observação de novas dimensões contribuirá para o aperfeiçoamento das estatísticas vitais. 


\section{Colaboradores}

MOA Ramalho e PG Frias participaram de todas as etapas da construção deste artigo; LCM Vanderlei participou da revisão crítica relevante ao conteúdo intelectual e aprovação final da versão a ser publicada. VC Macêdo participou da interpretação dos dados, redação do artigo e aprovação final da versão a ser publicada. PIC Lira participou da análise, interpretação dos dados e aprovação final da versão a ser publicada.

\section{Referências}

1. Mello-Jorge MHP, Laurenti R, Gotlieb SLD. Avaliação da qualidade das estatísticas vitais brasileiras: a experiência da implementação do SIM e SINASC. Cien Saude Colet 2007; 12(3):643-654.

2. Lima CRA, Schramm JMA, Coeli M, Silva MEM. Revisão das dimensões de qualidade dos dados e métodos aplicados na avaliação dos sistemas de informação em saúde. Cad Saude Publica 2009; 25(10):2095-2109.

3. Soares JAPS, Horta FMB, Caldeira AP. Avaliação da qualidade das informações em declarações de óbitos infantis. Rev Bras Saude Mater Infant 2007; 7(3):289295.

4. Frias PG, Szwarcwald CL, Lira PIC. Estimação da mortalidade infantil no contexto de descentralização do Sistema Único de Saúde (SUS). Rev Bras Saude Mater Infant 2011; 11(4):463-470.

5. Felix JD, Zandonade E, Amorim MHC, Castro DS. Avaliação da completude das variáveis epidemiológicas do Sistema de Informação sobre Mortalidade em mulheres com óbitos por câncer de mama na Região Sudeste - Brasil (1998 a 2007). Cien Saude Colet 2012; 17(4):945-953.

6. Szwarcwald CL, Leal MC, Andrade CLT, Souza Jr. PRB. Estimação da mortalidade infantil no Brasil: o que dizem as informações sobre óbitos e nascimentos do Ministério da Saúde? Cad Saude Publica 2002; 18(6):17251736.

7. Andrade CLT, Szwarcwald CL. Desigualdades sócio-espaciais da adequação das informações de nascimentos e óbitos do Ministério da Saúde, Brasil, 2000-2002. Cad Saude Publica 2007; 23(5):1207-1216.

8. França E, Abreu DX, Rao C, Lopez AD. Evaluation of cause-of-death statistics for Brazil, 2002-2004. Int J Epidemiol 2008; 37(4):891-901.

9. Schmidt MI, Duncan BB, Silva GA, Menezes AM, Monteiro CA, Barreto SM, Chor D, Menezes PR. Chronic non communicable diseases in Brazil: burden and current challenges. The Lancet [serial on the Internet] 2011 May [cited 2014 Jan 8]. Available from: http://www. thelancet.com

10. Santa Helena ET, Rosa MB. Avaliação da qualidade das informações relativas aos óbitos em menores de um ano Blumenau, 1998. Rev Bras Saude Mater Infant 2003; 3(1):75-83

11. Vanderlei LC, Arruda BKG, Frias PG, Arruda S. Avaliação da Qualidade de Preenchimento das Declarações de Óbito em Unidade Terciária de Atenção à Saúde Materno-Infantil. Inf Epidemiol SUS 2002; 11(1)7-14.

12. Szwarcwald, CL. Morais Neto OL, Frias PG, Souza Junior PRB, Escalante JJC, Lima RB, Viola RC. Busca ativa de óbitos e nascimentos no Nordeste e na Amazônia Legal: estimação das coberturas do SIM e do Sinasc nos municípios brasileiros. Brasil: Saúde Brasil 2011. Uma análise da situação de saúde; 2011. p. 79-97.

13. Frias PG, Szwarcwald CL, Souza Junior PRB, Almeida WS, Lira PIC. Correção de informações vitais: estimação da mortalidade infantil, Brasil, 2000-2009. Rev Saude Publica 2013; 47(6):1048-1058.

14. Frias PG, Pereira PMH, Andrade CLT, Szwarcwald CL. Sistema de Informações sobre Mortalidade: estudo de caso em municípios com precariedade dos dados. Cad Saude Publica 2008; 24(10):2257-2266. 
15. Rodrigues M, Bonfim C, Frias PG, Braga C, Gurgel IGD, Costa TR, Medeiros Z. Análise espacial da mortalidade infantil e adequação das informações vitais: uma proposta para definição de áreas prioritárias. Cien Saude Colet 2013; 19(7):2047-2054.

16. Vanderlei LCM, Navarrete MLV. Mortalidade infantil evitável e barreiras de acesso à atenção básica no Recife, Brasil. Rev Saude Publ 2013; 47(2):379-389.

17. Rodrigues M, Bonfim C, Frias PG, Braga C, Gurgel IGD, Medeiros Z. Differentials in vital information in the state of Pernambuco, Brazil, 2006-2008. Rev bras Epidemiol [serial on the Internet] 2012 June [cited 2013 June 24]:15(2):[about 9 p.]. Available from: http://www.scielo.br/scielo.php?script=sci_arttext\&pi$\mathrm{d}=$ S1415-790X2012000200005\&lng=en

18. Brasil. Ministério da Saúde (MS). Datasus. [site da Internet]. [acessado 2014 jan 8]. Disponível em: http:// msbbs.datasus.gov.br/public/default.html

19. Instituto Brasileiro de Geografia e Estatística (IBGE). Censo Demográfico. Rio de Janeiro: IBGE; 2010.

20. Romero DE, Cunha CB. Avaliação da qualidade das variáveis sócio-econômicas e demográficas dos óbitos de crianças menores de um ano registrados no Sistema de Informações sobre mortalidade do Brasil (1996/2001). Cad Saude Publica 2006; 22(3):673-684.

21. Mendes ACG, Lima MM, Sá DA, Oliveira LCS, Maia LTS. Uso da metodologia de relacionamento de bases de dados para qualificação da informação sobre mortalidade infantil nos municípios de Pernambuco. Rev Bras Saude Mater Infant 2012; 12(3):243-249.

22. Costa JMBS, Frias PG. Avaliação da completitude das variáveis da declaração de óbitos de menores de um ano residentes em Pernambuco,1997-2005. Cien Saude Colet 2011; 16(Supl. 1):1267-1274.

23. Barbuscia DM, Rodrigues-Júnior AL. Completude da informação nas Declarações de Nascido Vivo e nas Declarações de Óbito, neonatal precoce e fetal, da região de Ribeirão Preto, São Paulo, Brasil, 2000-2007. Cad Saude Publica 2011; 27(6):1192-1200.
24. Pedrosa LDCO, Sarinho WS, Ximenes RAA, Ordonha MR. Qualidade dos dados sobre óbitos neonatais precoces. Rev Ass Med Bras 2007; 53(5):389-394.

25. Figueiroa BQ, Vanderlei LCM, Frias PG, Carvalho PI, Szwarcwald CL. Análise da cobertura do Sistema de Informações sobre Mortalidade em Olinda, Pernambuco, Brasil. Cad Saude Publica 2013; 29(3):475-484.

26. Haraki CAC, Gotlieb SLD, Laurenti R. Confiabilidade do Sistema de Informações sobre Mortalidade em município do sul do Estado de São Paulo. Rev bras epidemiol 2005; 8(1):19-24.

27. Stuque CO, Cordeiro JA, Cury PM. Avaliação dos erros ou falhas de preenchimento dos atestados de óbitos feitos pelos clínicos e pelos patologistas. J Bras de Patol Med Lab 2003; 39(4):361-364.

28. Niobey FML, Cascão AM, Duchiade MP, Sabroza PC. Qualidade do preenchimento de atestados de óbitos de menores de um ano na região metropolitana do Rio de Janeiro. Rev Saude Publica 1990; 24(4):311-318.

29. Caetano SF, Vanderlei LC, Frias PG. Avaliação da completitude dos Instrumentos de Investigação do Óbito Infantil no município de Arapiraca, Alagoas. Cad Saude Colet 2013; 21(3):309-317.

30. Mathias TAF, Assunção NA, Silva GF. Óbitos infantis investigados pelo Comitê de Prevenção da Mortalidade Infantil em região do Estado do Paraná. Rev Esc Enferm USP 2008; 42(3):445-453.

31. Brasil. Ministério da Saúde (MS). Secretaria de Vigilância em Saúde. Painel de Monitoramento da Mortalidade Infantil e Fetal. [site da Internet]. [acessado 2014 jan 8]. Disponível em: http://svs.aids.gov.br/dashboard/ mortalidade/infantil.show.mtw

Artigo apresentado em 09/07/2014

Aprovado em 29/11/2014

Versão final apresentada em 01/12/2014 